ARTICLE

Received 15 Jun 2014 | Accepted 18 Jul 2014 | Published 27 Aug 2014

DOl: $10.1038 /$ ncomms5739

OPEN

\title{
Multiple intrinsically identical single-photon emitters in the solid state
}

\author{
L.J. Rogers' ${ }^{1}$, K.D. Jahnke ${ }^{1}$, T. Teraji ${ }^{2}$, L. Marseglia ${ }^{1}$, C. Müller ${ }^{1}$, B. Naydenov ${ }^{1}$, H. Schauffert ${ }^{1}$, C. Kranz ${ }^{3}$, \\ J. Isoya ${ }^{4}$, L.P. McGuinness ${ }^{1} \&$ F. Jelezko ${ }^{1}$
}

Emitters of indistinguishable single photons are crucial for the growing field of quantum technologies. To realize scalability and increase the complexity of quantum optics technologies, multiple independent yet identical single-photon emitters are required. However, typical solid-state single-photon sources are inherently dissimilar, necessitating the use of electrical feedback or optical cavities to improve spectral overlap between distinct emitters. Here we demonstrate bright silicon vacancy $\left(\mathrm{SiV}^{-}\right)$centres in low-strain bulk diamond, which show spectral overlap of up to $91 \%$ and nearly transform-limited excitation linewidths. This is the first time that distinct single-photon emitters in the solid state have shown intrinsically identical spectral properties. Our results have impact on the application of single-photon sources for quantum optics and cryptography.

\footnotetext{
${ }^{1}$ Institute for Quantum Optics and Center for Integrated Quantum Science and Technology (IQ ${ }^{\text {st }}$ ), Ulm University, Albert-Einstein-Allee 11, Ulm D-89081, Germany. ${ }^{2}$ National Institute for Materials Science, 1-1 Namiki, Tsukuba, Ibaraki 305-0044, Japan. ${ }^{3}$ Institute of Analytical and Bioanalytical Chemistry, Ulm University, Ulm D-89081, Germany. ${ }^{4}$ Research Center for Knowledge Communities, University of Tsukuba, 1-2 Kasuga, Tsukuba, Ibaraki 305-8550, Japan. Correspondence and requests for materials should be addressed to L.J.R. (email: lachlan.j.rogers@quantum.diamonds).
} 
S ingle transform-limited photons are an essential resource ${ }^{1-3}$ for many quantum interference experiments, as indistinguishability between photons allows path-oforigin information to be erased. This makes it possible to investigate fundamental quantum optics phenomena, which have applications in quantum imaging ${ }^{4}$, quantum computing ${ }^{1}$ and quantum repeaters ${ }^{2}$. Interactions between indistinguishable photons from multiple emitters can also be used to create entangled quantum states over macroscopic physical distances ${ }^{3}$. However, to date, it has been proven difficult to achieve indistinguishability between distinct single-photon sources.

Individual trapped ions in vacuum are a natural source of identical photons, limited primarily by their independent motion $^{5}$. The solid-state environment overcomes this limitation and allows distinct emitters to be addressed, but also adds complexity and local variations to the emitter environment. It has been necessary to use optical cavities ${ }^{6}$ and/or electrical tuning ${ }^{7-9}$ to interfere photons from distinct emitters ${ }^{10}$ in the solid state. Quantum dots and single molecules have demonstrated transform-limited linewidths over short timescales, allowing interference between photons from the same emitter 6,11 and physically separated emitters ${ }^{7}$. Colour centres in diamond, located deep within an ultrapure lattice, show excellent photostability and are attractive single-photon sources. The negative nitrogen vacancy $\left(\mathrm{NV}^{-}\right)$centre is well studied and has been used for early photonics applications ${ }^{12,13}$, but its broad spectral emission is ill-suited to single-photon technologies ${ }^{3}$.

The negative silicon vacancy $\left(\mathrm{SiV}^{-}\right)$centre in diamond has promising spectral properties ${ }^{14-16}$. It has a strong optical transition with a prominent zero-phonon line (ZPL) at $737 \mathrm{~nm}$ and only a weak phonon sideband ${ }^{17-21}$. Structurally, it comprises a silicon atom located between adjacent vacancies in the diamond lattice $^{15,22,23}$ (Fig. 1a). The strong ZPL has sparked interest, but large variation in spectral properties between individual sites ${ }^{16}$ has limited the value of $\mathrm{SiV}^{-}$as a single-photon emitter. Here we obtain highly uniform, narrow linewidths from single $\mathrm{SiV}^{-}$ centres in a low-strain bulk diamond.

\section{Results}

Lifetime-limited flourescence linewidth. The diamond sample was prepared on a high-pressure high-temperature diamond substrate, as this fabrication technique can produce high crystalline quality with low strain. A layer of ultrapure diamond was overgrown on this $\langle 001\rangle$-oriented substrate with chemical vapour deposition, allowing precise control of impurity concentrations. Silicon was introduced to the plasma and incorporated into the diamond at concentrations below 1 p.p.b. (see Methods). Single $\mathrm{SiV}^{-}$defects formed during growth were observed by fluorescence confocal microscopy (Fig. 1b,c). Photon antibunching measurements provided confirmation of singlephoton emission.

At $4 \mathrm{~K}$, the ZPL consists of four lines as a result of optically allowed transitions between doublet ground and excited states $^{24,25}$ (Fig. 2a,b). Each of these transitions was resonantly excited and fluorescence was detected in the sideband. Scanning the laser frequency produced photoluminescence excitation (PLE) spectra, shown in Fig. 2c for a single emitter.

The pair of lines at shorter wavelengths (higher energy), here labelled lines A and B, had full-width at half maximum (FWHM) of 352 and $409 \mathrm{MHz}$. The pair of lines at longer wavelengths were considerably narrower at $136 \mathrm{MHz}$ (line C) and $119 \mathrm{MHz}$ (line D). All of these PLE spectra were recorded at minimal laser intensities to avoid power broadening. To compare these linewidths with their expected Fourier-transform limit, pulsed $532 \mathrm{~nm}$ excitation was used to measure the excited-state lifetime for several individual $\mathrm{SiV}^{-}$centres (Fig. 2c). A decay time of $1.72 \pm 0.04 \mathrm{~ns}$ was measured at $4 \mathrm{~K}$. Our measured result for line $\mathrm{D}$ is therefore only $26 \%$ over the transform-limited linewidth of $94 \mathrm{MHz}$.

Spectral stability of $\mathrm{SiV}$ emitters. At $4 \mathrm{~K}, 71 \%$ of the total ZPL flourescence is contained in line C. Combined with the DebyeWaller factor of $70 \%{ }^{26}$, this means that half of the total $\mathrm{SiV}^{-}$ flourescence is emitted into the almost transform-limited line C. In addition, this transition is known to arise from a single (axial) dipole moment ${ }^{24,25}$. These properties are ideal for coupling to narrowband cavities and waveguides, and for single-photon interference experiments, including quantum cryptography. The following discussion therefore focusses on line C.

Over a period of $7 \mathrm{~h}$, the line position was recorded for a single $\mathrm{SiV}^{-}$centre (Fig. 3a). The variation in line position was found to be $\pm 4 \mathrm{MHz}$, which is within the $95 \%$ confidence interval for this parameter obtained from a Lorentzian fit. The fact that we observe no spectral diffusion highlights the ability of $\mathrm{SiV}^{-}$to produce indistinguishable photons over long periods of time. No blinking was observed for any of the $\mathrm{SiV}^{-}$sites measured in this study.
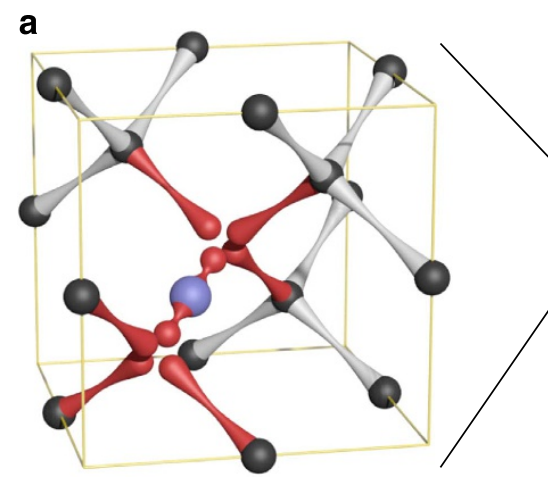

b
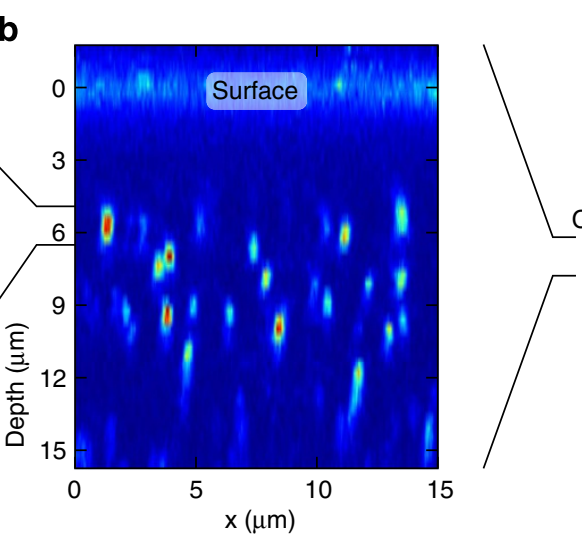

Figure 1 | Silicon vacancy centres in diamond. (a) Physical structure of the SiV ${ }^{-}$centre in the diamond lattice, with a silicon atom lying in between adjacent vacancies. (b) Fluorescence confocal image showing the depth profile of single SiV ${ }^{-}$centres beneath the diamond surface (corrected for refractive index). (c) The $\mathrm{SiV}^{-}$centres were present in a single crystal chemical vapour deposition (CVD) diamond layer, and had been incorporated during growth due to silicon doping of the plasma. The CVD layer was grown homoepitaxially on a low strain, high-pressure high-temperature (HPHT) diamond substrate and investigated from the top. 
a

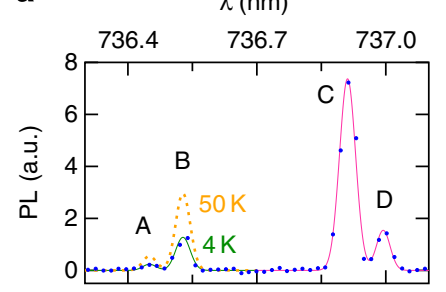

b

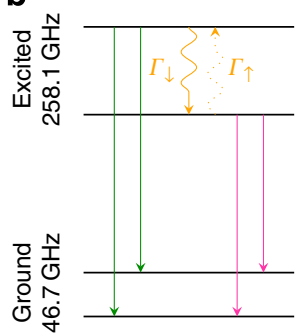

d

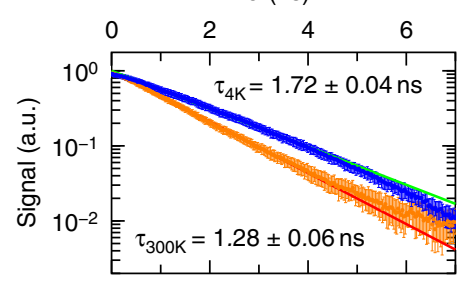

C

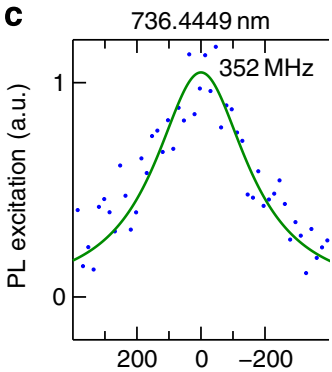

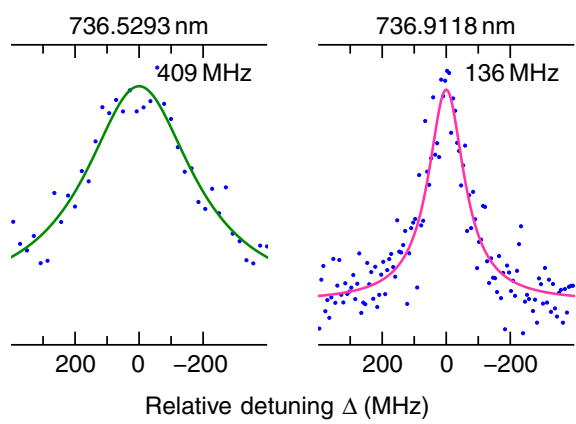

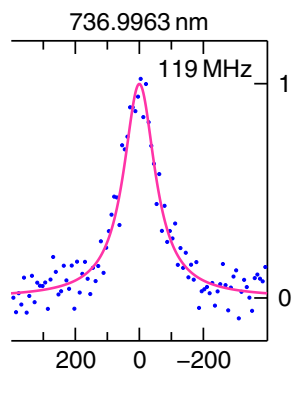

Figure 2 | Linewidths near the transform limit. (a) The SiV ${ }^{-}$ZPL has a four-line fine structure (linewidth limited here by spectrometer resolution). The left-hand pair of lines are a mirror image of the right-hand pair, but they have much lower intensity at $4 \mathrm{~K}$. These higher energy lines regain intensity with increasing temperature, and the dotted line illustrates the relative intensity at $50 \mathrm{~K}$. (b) This fine structure arises from transitions between a doublet excited state and a doublet ground state that both have zero-field splittings. Population is exchanged between the two excited state branches, but at low temperature the upward rate $\Gamma_{\uparrow}$ is slow relative to the state lifetime. The downward exchange rate $\Gamma_{\downarrow}$ dominates the relaxation within the excited state, which accounts for the loss of emission intensity for the high-energy pair of lines. (c) Excitation spectra of the four lines that comprise the $\mathrm{SiV}^{-}$ZPL (amplitudes normalized). The higher energy lines are wider, consistent with a shorter effective lifetime due to thermalization between the two excited state branches. (d) The radiative decay lifetime of $\mathrm{SiV}^{-}$increases from $1.28 \mathrm{~ns}$ at room temperature to $1.72 \mathrm{~ns}$ at $4 \mathrm{~K}$, corresponding to a transform-limited PLE linewidth of $94 \mathrm{MHz}$. The error bars indicate s.d. across 15 randomly selected SiV ${ }^{-}$centres.

PLE spectra were recorded for all 20 clearly resolvable $\mathrm{SiV}^{-}$ sites in an arbitrary scan region, shown in Fig. 3 b. On a $\{001\}$ surface, the projections of $\langle 111\rangle$-aligned $\mathrm{SiV}^{-}$centres form two orthogonal sets ${ }^{24}$. Scanning with two orthogonal laser polarizations (encoded in colour) revealed the set to which each site in the region belonged ${ }^{24}$. The line position for each site is illustrated in Fig. 3c. Within each orientation set the sites are closely spaced, although the distinct orientations are separated by about $5 \mathrm{GHz}$. Figure $3 \mathrm{~d}$ shows a histogram of the shifts between consecutive sites. Out of the 20 centres, 11 pairs of $\mathrm{SiV}^{-}$have separations $<1$ transform-limited FWHM. This means that for a randomly chosen site there is $>50 \%$ probability of finding a second site in this scan region whose line is displaced by $<1$ FWHM. The two closest sites had lines separated by only $6 \mathrm{MHz}$ (within the confidence interval of the fit), meaning a spectral overlap of at least $91 \%$. Notably, this spectral overlap was achieved without external tuning of the spectral position.

$\mathrm{SiV}$ incorporation in solid immersion lens. An advantage to solid-state emitters over trapped ions is their reliable addressing, which allows direct incorporation into photonic and plasmonic devices. $\mathrm{SiV}^{-}$ensembles have been coupled into cavities ${ }^{27}$, and here we incorporate single $\mathrm{SiV}^{-}$centres into a solid-immersion lens (SILs) to demonstrate their readiness for applications (Fig. 4a). SILs allow increased collection of flourescence from high refractive index materials ${ }^{28,29}$, which is particularly useful during cryogenic experiments. Well-coupled $\mathrm{SiV}^{-}$centres were identified by scanning the depth profile with a confocal microscope. The SIL enhanced flourescence collection by about a factor of ten, giving a saturation flourescence up to $I_{\text {sat }}=730 \mathrm{kcs}^{-1}$. Using an oil-immersion objective on a flat surface, $\mathrm{SiV}^{-}$produced $I_{\text {sat }}=200 \mathrm{kcs}^{-1}$, which is comparable to a single $\mathrm{NV}^{-}$centre or bright molecule under the same conditions (Fig. 4b).

\section{Discussion}

Although narrow spectral lines were measured for the lowerenergy transitions, the higher energy pair of lines were broader by nearly a factor of three. It is important to account for this extra width of lines $\mathrm{A}$ and $\mathrm{B}$, and further information is provided by photoluminescence measured with a spectrometer. At $4 \mathrm{~K}$, lines $\mathrm{C}$ and $\mathrm{D}$ are much brighter than the higher-energy lines (Fig. 2a), which correspond to transitions from the upper branch of the excited state (Fig. 2b). With increasing temperature, the highenergy lines gain relative intensity, indicating that thermal relaxation occurs in the $\mathrm{SiV}^{-}$excited state. The downward exchange rate $\Gamma_{\downarrow}$ adds to the rate of decay to the ground state and reduces the effective lifetime of the upper branch. Consequently, lines $\mathrm{A}$ and $\mathrm{B}$ are broadened and lose intensity in photoluminescence. The upward exchange rate $\Gamma_{\uparrow}=\Gamma_{\downarrow} \exp \frac{\Delta E}{k_{\mathrm{B}} t}$ depends on the Boltzmann factor, making it small but still measurable at $4 \mathrm{~K}$. This additional rate out of the lower branch accounts for half of the exta linewidth above the transform limit for lines $\mathrm{C}$ and $\mathrm{D}$.

To explain the observed homogeneity between distinct centres, we reconsider the energy level scheme in Fig. $2 \mathrm{~b}$. The ground and excited states have E symmetry ${ }^{15,24,25}$ and are split due to spinorbit interaction ${ }^{24}$. In general, strain and electric fields can perturb these states to result in line shifts and increased splittings. Electric fields may be produced by nearby charged impurities and, therefore, vary across small spatial scales. The precise correspondence between orientation and line position for each 

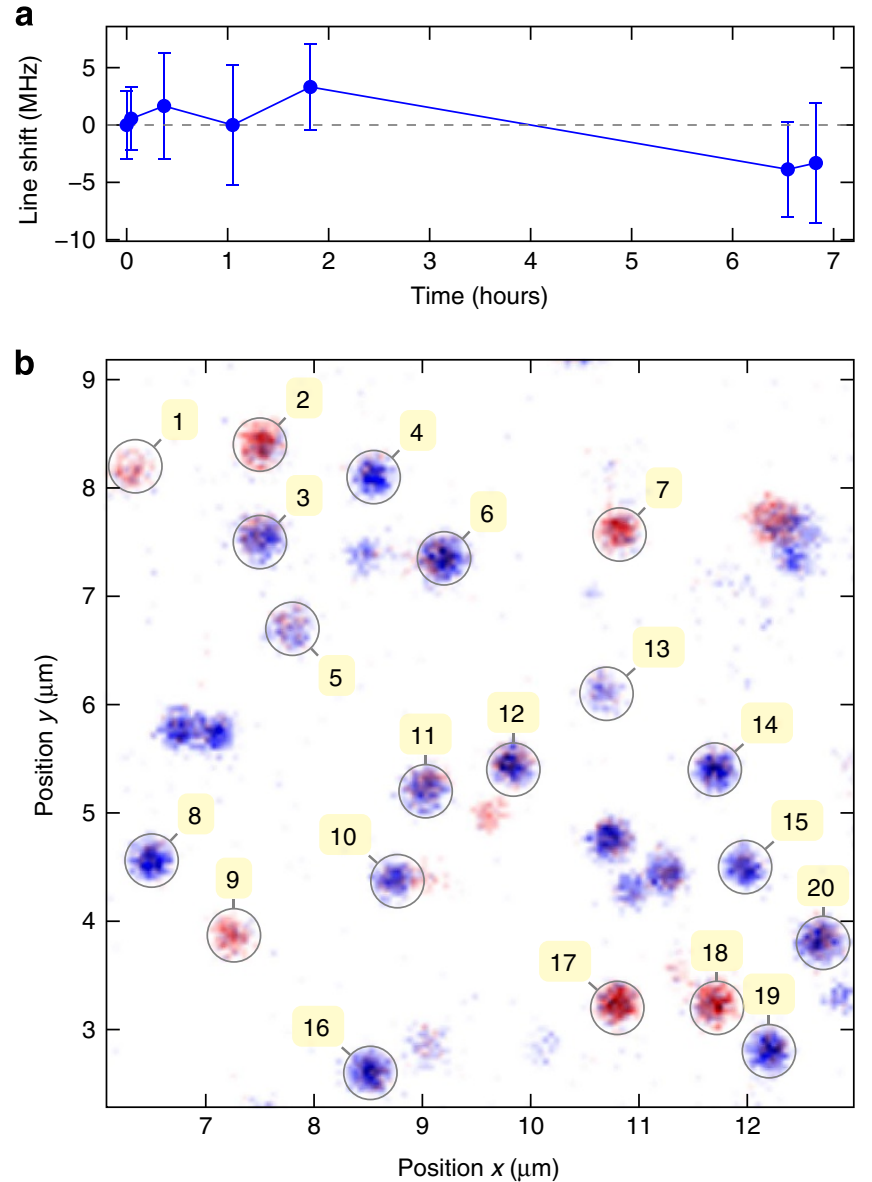

C

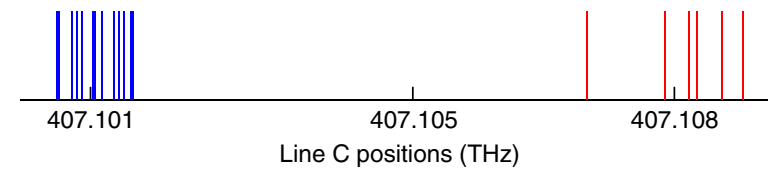

d

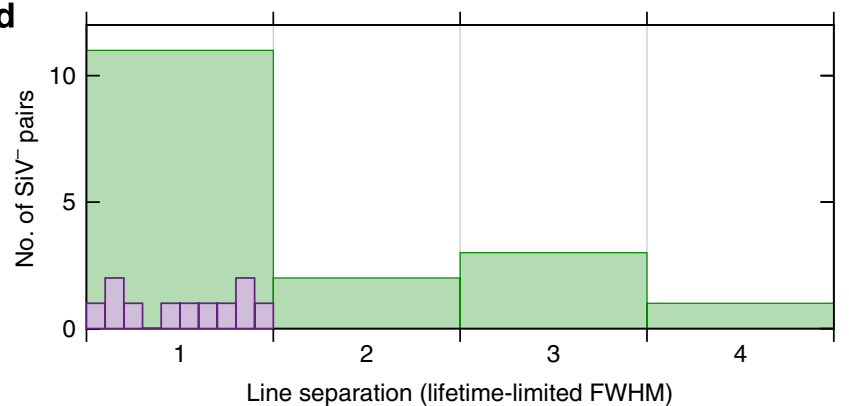

Figure 3 | Stability and uniformity of single SiV ${ }^{-}$centres. (a) No measurable spectral diffusion was detected over a period of $7 \mathrm{~h}$. Error bars represent a 95\% confidence interval for the line position extracted from a Lorentzian fit. (b) PLE spectra were measured for each of the 20 resolvable single $\mathrm{SiV}^{-}$centres in this confocal image (numbered). The image is a composite of two scans performed at orthogonal excitation polarizations, and so the apparent colour of the sites indicates its orientation in the crystal lattice. (c) Position of line C for each of the 20 $\mathrm{SiV}^{-}$centres. (d) Histogram of the shift between adjacent sites in $\mathbf{c}$. The primary bins are $94 \mathrm{MHz}$ wide corresponding to the transform-limited linewidth, and the composition of the first bin is illustrated in ten sub-bins. Eleven $\mathrm{SiV}^{-}$pairs had separations $<94 \mathrm{MHz}$, and four pairs had separations $<30 \%$ of the lifetime-limited linewidth. a
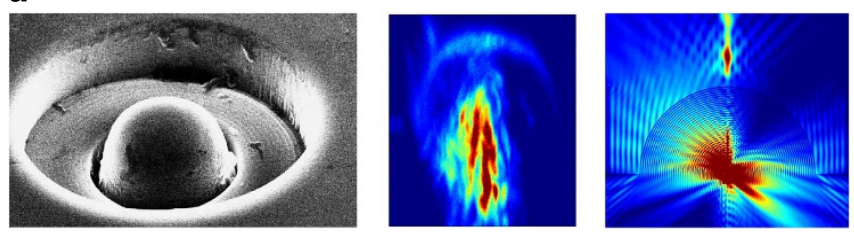

b

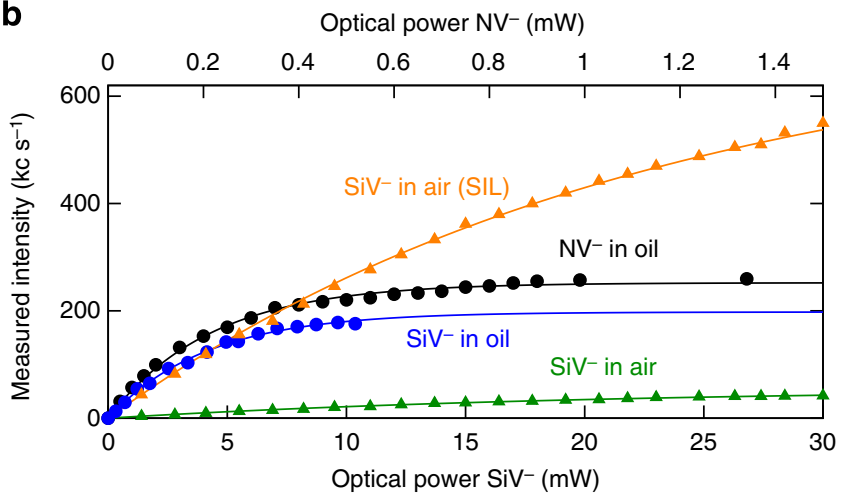

Figure 4 | SiV ${ }^{-}$incorporation into a SIL. (a) Scanning electron microscope image of a single SIL fabricated in the diamond surface. The vertical cross-section confocal image (centre) shows an intensity profile in the SIL with enhanced fluorescence detection for $\mathrm{SiV}^{-}$centres near the focus. This is in qualitative agreement with the simulated emission intensity (right) for a single dipole located at the centre of the solid immersion lens (calculated with finite-difference time-domain numerical package). (b) Using an air objective (numerical aperture $(N A)=0.95$ ), the saturation count rate $I_{\text {sat }}$ for $\mathrm{SiV}^{-}$under a SIL $\left(730 \mathrm{kc} \mathrm{s}^{-1}\right)$ is enhanced by more than a factor of 10 compared with an equivalent single $\mathrm{SiV}^{-}$ under a planar surface nearby $\left(I_{\text {sat }}=56 \mathrm{kc} \mathrm{s}^{-1}\right)$. To more easily compare $\mathrm{SiV}^{-}$with other emitters, a high performance $(\mathrm{NA}=1.35)$ oil-immersion objective was used at room temperature. SiV ${ }^{-}$sites show $I_{\text {sat }}=200 \mathrm{kc} \mathrm{s}^{-1}$, which is comparable to single $\mathrm{NV}^{-}$centres $\left(I_{\text {sat }}=252 \mathrm{kc} \mathrm{s}^{-1}\right)$.

$\mathrm{SiV}^{-}$measured here suggests that strain, which can be nearly uniform over a $7 \times 7-\mu \mathrm{m}$ region, is more influential than electric fields.

The large spin-orbit splittings of $46.7 \mathrm{GHz}(258.1 \mathrm{GHz})$ in the ground (excited) state help to make $\mathrm{SiV}^{-}$unresponsive to small transverse strains. This occurs because the effect of such strain is a small perturbation until strain splitting increases to about the magnitude of spin-orbit. We observed this effect in the groundstate splitting measured between lines $C$ and $D$, which varied much less $( \pm 1 \mathrm{GHz})$ than line position across the 20 sites and showed no correlation with orientation. This implies that the observed line shift results from axial strain. The inversion symmetry of $\mathrm{SiV}^{-}$(refs 15,24) reduces the influence of small axial strain, as inverting the strain direction does not change line shift. Our observations indicate that this shielding has a lower threshold than provided by spin-orbit for transverse strain. Despite the presence of residual strain in this sample region we were still able to find identical emitters.

The spectral properties we have presented establish $\mathrm{SiV}^{-}$as an attractive single-photon emitter. For such applications, it is usually of interest to have a high quantum yield, meaning that the probability of photon emission is high for each excitation event. Measuring the absolute quantum yield is difficult, because it is not possible to collect all of the photons emitted by a colour centre. Here we have reported saturation fluorescence, but the presence of poorly understood metastable states ${ }^{30}$ prevents a deduction of absolute quantum yield for $\mathrm{SiV}^{-}$from these data. It seems, 
however, that $\mathrm{SiV}^{-}$has a lower quantum yield than $\mathrm{NV}^{-}$, which has similar saturation flourescence but a longer excited-state lifetime of $13 \mathrm{~ns}^{12}$. We found the $\mathrm{SiV}^{-}$decay lifetime to be shorter $(1.28 \pm 0.06 \mathrm{~ns})$ at room temperature than at $4 \mathrm{~K}$ (Fig. $2 \mathrm{~d}$ ). This is consistent with a thermally activated non-radiative decay path ${ }^{17,26,31}$ and indicates an improvement of the $\mathrm{SiV}^{-}$quantum yield at low temperature. It may be possible to disable this non-radiative decay path and dramatically improve the quantum yield. This could explain how $\mathrm{SiV}^{-}$centres in nanodiamonds grown on iridium were able to produce flourescence rates up to $6.2 \mathrm{Mcs}^{-1}$ (ref. 32).

Another nuance in interpreting saturation flourescence is that photons are measured across the entire emission band of a colour centre (including the phonon sideband). For sources of indistinguishable single photons, the absolute quantum yield is less important than the 'effective' yield of photons in the desired transform-limited spectral line. The strong $\mathrm{ZPL}$ of $\mathrm{SiV}^{-}$gives it a significant advantage in this respect, and here we have established that line C can contain up to $50 \%$ of the total flourescence. Our results show that a single $\mathrm{SiV}^{-}$centre can provide indistinguishable photons at a collectable rate on the order of hundreds of kc s${ }^{-1}$.

In summary, we have demonstrated a uniform single-photon source in the solid state without requiring external tuning of the optical properties. We observe nearly transform-limited linewidths, without spectral diffusion, which would allow high spectral overlap between single photons emitted from distinct sources. The production of multiple, independent single-photon emitters with identical properties is essential to the scalability of a number of schemes that use entangled photons, including quantum computing with linear optics, and is expected to form a fundamental resource in quantum optics technologies. The $\mathrm{SiV}^{-}$centre is therefore promising for such applications.

\section{Methods}

Sample preparation. A $\langle 001\rangle$-oriented plate cut from a low-strain, type-IIa high-pressure high-temperature crystal (Sumitomo Electric Industries, Ltd) was used as a substrate for microwave plasma chemical vapour deposition. High-purity $\mathrm{H}_{2}$ and $\mathrm{CH}_{4}$ source gas, specified to $99.999 \%{ }^{12} \mathrm{C}$ isotopic enrichment (Cambridge Isotope Laboratories CLM-392) was used to produce the plasma. The residual $\mathrm{N}_{2}$ concentration was $<0.1$ p.p.b. for $\mathrm{H}_{2}$ and $<1$ p.p.b. for $\mathrm{CH}_{4}$. The total gas pressure, microwave power, methane concentration ratio $\left(\mathrm{CH}_{4} / \mathrm{H}_{2}\right)$, growth duration and substrate temperature employed were 120 Torr, $1.4 \mathrm{~kW}, 4 \%, 24 \mathrm{~h}$ and $950-1,000^{\circ} \mathrm{C}$, respectively. The homoepitaxial layer thickness estimated from the weight difference between initial substrate and after the growth was $\sim 60 \mu \mathrm{m}$ giving a growth rate of $2.4 \mu \mathrm{m} \mathrm{h}^{-1}$.

Cathodoluminescence spectra $\left(15 \mathrm{kV}\right.$ acceleration voltage, $2 \times 10^{-7} \mathrm{~A}$ incident beam current) taken at room temperature in a wavelength range from 200 to $800 \mathrm{~nm}$ provided information on the crystalline quality and optically active impurities. Emission from free-exciton recombination was observed from most of the growth surface, in addition to a weak signal at $738 \mathrm{~nm}$, which is assigned to $\mathrm{SiV}^{-}$. The reaction vessel of microwave plasma chemical vapour deposition contains mainly stainless steel and molybdenum. Quartz glass $\left(\mathrm{SiO}_{2}\right)$ was used for the windows of the vessel. When homoepitaxial film was grown under low microwave power, no $\mathrm{SiV}^{-}$fluorescence was observed. Increasing the microwave input power caused the plasma to extend and etch material from the quartz, picking up silicon. In this study, a $6 \mathrm{H}-\mathrm{SiC}$ single-crystal plate was also used as a silicon source to allow increased doping of grown diamond. The SiC plate was inserted between the diamond substrate and a molybdenum sample holder. It was possible to incorporate $\mathrm{SiV}^{-}$during growth at concentrations below $0.2 \mu \mathrm{m}^{3}$ $\left(\sim 1 \times 10^{-3}\right.$ p.p.b.). Incorporation of silicon occurred relatively uniformly over the whole lateral direction.

Optical measurements. The sample was mounted in a continuous flow helium cryostat capable of cooling to $4 \mathrm{~K}$. Single $\mathrm{SiV}^{-}$centres were imaged using a homebuilt confocal microscope. The excitation laser beam $(532 \mathrm{~nm})$ was focused onto the diamond surface through a 0.95 -numerical aperture microscope objective (or 1.35 numerical aperture oil-immersion objective for room temperature measurements). The objective was scanned to produce confocal images of sample regions. Fluorescence was collected by the same objective, filtered with a 725- to 775-nm band-pass filter and focused through a $25-\mu \mathrm{m}$ pinhole before detection on an avalanche photodiode. After the pinhole, the flourescence could also be sent to a spectrometer to acquire spectra from single sites. Using a high-resolution grating (1,596 grooves per $\mathrm{mm}$ ) allowed the 4-four component fine structure to be resolved.

PLE measurements were performed on the same setup, but using a

Titanium:Sapphire laser with $50 \mathrm{kHz}$ linewidth for excitation. This laser could scan across the entire ZPL and detection was performed on the sideband by switching the filter to a 750 - to 810 -nm band-pass.

Decay lifetime of the excited state was measured by changing the excitation laser to a Titanium:Sapphire pumped optical parametric oscillator, producing $50 \mathrm{fs}$ pulses at $80 \mathrm{MHz}$ repetition rate. This optical parametric oscillator was set to $532 \mathrm{~nm}$ to excite $\mathrm{SiV}^{-}$off-resonantly. The avalanche photodiode signal was analysed using a PicoQuant TimeHarp counting card, with a resolution of 29 ps.

SIL fabrication. SILs were produced in a region of the sample known to contain a medium density of $\mathrm{SiV}^{-}$centres at a depth of $2.5-6 \mu \mathrm{m}$ below the surface. The site density was high enough to assure a reasonable probability of coupling a SIL to a colour centre. The fabrication process was performed with a Helios Nanolab 600 Focused Ion Beam lithography system. Each SIL had a radius of $7 \mu \mathrm{m}$ and a depth of $7 \mu \mathrm{m}$. The SIL images were taken with a Helios Nanolab 600 Focused Ion Beam lithography system.

\section{References}

1. Knill, E., Laflamme, R. \& Milburn, G. J. A scheme for efficient quantum computation with linear optics. Nature 409, 46-52 (2001).

2. Yuan, Z.-S. et al. Experimental demonstration of a BDCZ quantum repeater node. Nature 454, 1098-1101 (2008).

3. Bernien, H. et al. Heralded entanglement between solid-state qubits separated by three metres. Nature 497, 86-90 (2013).

4. Strekalov, D. V., Sergienko, A. V., Klyshko, D. N. \& Shih, Y. H. Observation of two-photon "ghost" interference and diffraction. Phys. Rev. Lett. 74, 3600-3603 (1995).

5. Beugnon, J. et al. Quantum interference between two single photons emitted by independently trapped atoms. Nature 440, 779-782 (2006).

6. Santori, C., Fattal, D., Vučković, J., Solomon, G. S. \& Yamamoto, Y. Indistinguishable photons from a single-photon device. Nature 419, 594-597 (2002).

7. Patel, R. B. et al. Two-photon interference of the emission from electrically tunable remote quantum dots. Nat. Photon. 4, 632-635 (2010).

8. Bernien, H. et al. Two-photon quantum interference from separate nitrogen vacancy centers in diamond. Phys. Rev. Lett. 108, 043604 (2012).

9. Sipahigil, A. et al. Quantum interference of single photons from remote nitrogen-vacancy centers in diamond. Phys. Rev. Lett. 108, 143601 (2012).

10. Shields, A. J. Semiconductor quantum light sources. Nat. Photon. 1, 215-223 (2007).

11. Kiraz, A. et al. Indistinguishable photons from a single molecule. Phys. Rev. Lett. 94, 223602 (2005).

12. Faraon, A., Santori, C., Huang, Z., Acosta, V. M. \& Beausoleil, R. G. Coupling of nitrogen-vacancy centers to photonic crystal cavities in monocrystalline diamond. Phys. Rev. Lett. 109, 033604 (2012).

13. Barclay, P. E., Fu, K.-M. C., Santori, C., Faraon, A. \& Beausoleil, R. G. Hybrid nanocavity resonant enhancement of color center emission in diamond. Phys. Rev. X 1, 011007 (2011).

14. Collins, A. T., Kamo, M. \& Sato, Y. A spectroscopic study of optical centers in diamond grown by microwave-assisted chemical vapor deposition. J. Mater. Res. 5, 2507-2514 (1990)

15. Goss, J. P., Jones, R., Breuer, S. J., Briddon, P. R. \& Öberg, S. The twelve-line $1.682 \mathrm{eV}$ luminescence center in diamond and the vacancy-silicon complex. Phys. Rev. Lett. 77, 3041-3044 (1996).

16. Neu, E. et al. Low-temperature investigations of single silicon vacancy colour centres in diamond. New J. Phys. 15, 043005 (2013).

17. Feng, T. \& Schwartz, B. D. Characteristics and origin of the $1.681 \mathrm{eV}$ luminescence center in chemical-vapor-deposited diamond films. J. Appl. Phys. 73, 1415-1425 (1993).

18. Brown, S. W. \& Rand, S. C. Site symmetry analysis of the $738 \mathrm{~nm}$ defect in diamond. J. Appl. Phys. 78, 4069-4075 (1995).

19. Clark, C. D., Kanda, H., Kiflawi, I. \& Sittas, G. Silicon defects in diamond. Phys Rev. B 51, 16681-16688 (1995).

20. Edmonds, A. M., Newton, M. E., Martineau, P. M., Twitchen, D. J. \& Williams, S. D. Electron paramagnetic resonance studies of silicon-related defects in diamond. Phys. Rev. B 77, 245205 (2008).

21. Neu, E. et al. Single photon emission from silicon-vacancy colour centres in chemical vapour deposition nano-diamonds on iridium. New J. Phys. 13, 025012 (2011).

22. Turukhin, A. V., Liu, C. -H., Gorokhovsky, A. A., Alfano, R. R. \& Phillips, W Picosecond photoluminescence decay of si-doped chemical-vapor-deposited diamond films. Phys. Rev. B 54, 16448-16451 (1996).

23. Wang, C., Kurtsiefer, C., Weinfurter, H. \& Burchard, B. Single photon emission from $\mathrm{SiV}$ centres in diamond produced by ion implantation. J. Phys. B Atom. Mol. Opt. Phys. 39, 37 (2006). 
24. Rogers, L. J. et al. Electronic structure of the negatively charged silicon-vacancy center in diamond. Phys. Rev. B 89, 235101 (2014).

25. Hepp, C. et al. Electronic structure of the silicon vacancy color center in diamond. Phys. Rev. Lett. 112, 036405 (2014).

26. Collins, A. T., Allers, L., Wort, C. J. \& Scarsbrook, G. A. The annealing of radiation damage in de Beers colourless CVD diamond. Diamond Related Mater. 3, 932-935 (1994).

27. Lee, J. C., Aharonovich, I., Magyar, A. P., Rol, F. \& Hu, E. L. Coupling of silicon-vacancy centers to a single crystal diamond cavity. Opt. Express $\mathbf{2 0}$, 8891-8897 (2012).

28. Marseglia, L. et al. Nanofabricated solid immersion lenses registered to single emitters in diamond. Appl. Phys. Lett. 98, 133107 (2011).

29. Castelletto, S. et al. Diamond-based structures to collect and guide light. New J. Phys. 13, 025020 (2011).

30. Neu, E. et al. Electronic transitions of single silicon vacancy centers in the nearinfrared spectral region. Phys. Rev. B 85, 245207 (2012).

31. Rogers, L. How far into the infrared can a colour centre in diamond emit? Phys. Proc. 3, 1557-1561 (2010).

32. Neu, E., Agio, M. \& Becher, C. Photophysics of single silicon vacancy centers in diamond: implications for single photon emission. Opt. Express 20, 19956-19971 (2012)

\section{Acknowledgements}

We acknowledge ERC, EU projects (SIQS, DIADEMS and EQUAM), DFG (FOR 1482, FOR 1493 and SFBTR 21), JST, BMBF, the Alexander von Humboldt, and the
Sino-German and Volkswagen foundations for funding. We acknowledge G. Neusser and the FIB Center UUlm for support in manufacturing SILs.

\section{Author contributions}

L.J.R. and K.D.J. did the low-temperature PLE experiments; T.T. and J.I. produced the sample; L.P.M., B.N. and C.M. characterized the sample at room temperature and performed saturation experiments; L.M. and C.K. fabricated and characterized the SILs; H.S performed the SIL simulations. The manuscript was written by L.J.R., K.D.J. and L.P.M. and all authors discussed the results and commented on the manuscript.

\section{Additional information}

Competing financial interests: The authors declare no competing financial interests.

Reprints and permission information is available online at http://npg.nature.com/ reprintsandpermissions/

How to cite this article: Rogers, L. J. et al. Multiple intrinsically identical single-photon emitters in the solid state. Nat. Commun. 5:4739 doi: 10.1038/ncomms5739 (2014).

(c) (1) (2) This work is licensed under a Creative Commons AttributionBY NG SA NonCommercial-ShareAlike 4.0 International License. The images or other third party material in this article are included in the article's Creative Commons license, unless indicated otherwise in the credit line; if the material is not included under the Creative Commons license, users will need to obtain permission from the license holder to reproduce the material. To view a copy of this license, visit http:// creativecommons.org/licenses/by-nc-sa/4.0/ 